\title{
FILTERING AND PREDICTING THE COST OF HIDDEN PERISHED ITEMS IN AN INVENTORY MODEL
}

\author{
LAKHDAR AGGOUN and LAKDERE BENKHEROUF \\ Sultan Qaboos University \\ Department of Mathematics and Statistics \\ P.O. Box 36, Al-Khod 123, Sultanate of Oman
}

(Received August 2001; Revised June 2002)

\begin{abstract}
This paper is concerned with a discrete time, discrete state inventory model for items of changing quality. Items are assumed to be in one of a finite number, $M$, of quality classes that are ordered in such a way that Class 1 contains the best quality and the last class contains the pre-perishable quality. The changes of items' quality are dependent on the state of the ambient environment. Furthermore, at each epoch time, items of different classes may be sold or moved to a lower quality class or stay in the same class. These items are priced according to their quality, and costs are incurred as items lose quality. Based on observing the history of the inventory level and prices, we propose recursive estimators as well as predictors for the joint distribution of the accumulated losses and the state of the environment.
\end{abstract}

Key words: Hidden Markov Models, Optimal Filtering, Inventory Control.

AMS subject classifications: 60K30, 60J10, 90B05.

\section{Introduction}

This paper is concerned with a discrete time, discrete state inventory model for items of changing quality (or aging like in [4]). However, in [4], movement between classes was deterministic, i.e. each item, at the end of each epoch, moved to an older category or class provided that it did not perish. In the present situation, items are allowed to remain within the same class for an indefinite length of time, with some probability of loss of quality, and move to a lower quality class (see dynamics equation (1.2), which is substantially different from dynamics equation (1.2) in [4]). Another new feature here is the quantification of the financial loss incurred by the loss of quality in the inventory (see equation (1.4) and Theorem 1). Finally, Theorem 2, which provides a one-step ahead recursive predictor, has no analog in [4].

Items are assumed to be in one of a finite number, $M$, of quality classes that are ordered in such a way that Class 1 contains the best quality and the last class contains the pre-perishable quality.

1. The vector $I_{n}=\left(I_{n}^{1}, \ldots, I_{n}^{M}\right)$ represents the inventory level at time $n$ where $I_{n}^{i}$ refers to the inventory level of Class $i$ at time $n, i=1, \ldots, M$ and $n=0,1, \ldots$.

2. At time $n$, items in Class $i$ are assumed to have a unit price $P_{n}^{i}$ such that $P_{n}^{i}=\theta_{i} p_{n}$ for some nonnegative real number $p_{n}$, with $\theta_{1}=1$ and $\theta_{1}>\theta_{2}>\ldots>\theta_{M}>0$. The latter condition reflects the order of the quality classes. We also assume that the price process $\left\{P_{n}\right\}$ defined by $P_{n}=\left(P_{n}^{1}, \ldots, P_{n}^{M}\right)$ is predicable with respect to the history of the inventory model. 
3. The random vector $D_{n}=\left(D_{n}^{1}, \ldots, D_{n}^{M}\right)$, with distribution $\Phi(\cdot)$ and marginals $\left\{\Psi_{n}^{i}\right\}_{n \in \mathbb{N}}, i=1, \ldots, M$, defined on $\mathbb{Z}_{+}^{M}$ represents the demand process at time $n$.

4. New arriving items are assigned to Class 1 and these are represented by a predictable process $\left\{U_{n}\right\}$ defined on $\mathbb{Z}_{+}$.

5. Define the operator " $\circ$ " such that for any nonnegative random variable $X$ and $\alpha \in(0,1)$,

$$
\alpha \circ X=\sum_{j=1}^{X} Z_{j}
$$

where $Z_{j}$ is a sequence of iid random variables, independent of $X$, such that

$$
P\left(Z_{j}=1\right)=1-P\left(Z_{j}=0\right)=\alpha .
$$

Also, let $\left\{X_{n}\right\}_{n \in \mathbb{N}}$ be a stochastic process with a finite state space $S_{X}$ of size $N$, which we identify without loss of generality with the set of unit vectors $\left\{e_{1}, \ldots, e_{N}\right\}$ in $\mathbb{R}^{N}$. The stochastic process $\left\{X_{n}\right\}_{n \in \mathbb{N}}$ represents the state of the ambient environment. We assume that at each time $n$, items in the stock may experience some kind of change of quality depending on the state of the environment $\left\{X_{n}\right\}$ where the stock is held and these changes follow the dynamics

$$
\begin{gathered}
I_{n}^{1}=F^{1}\left(X_{n-1}\right) \circ I_{n-1}^{1}-D_{n}^{1}+U_{n} \\
I_{n}^{i}=F^{i}\left(X_{n-1}\right) \circ I_{n-1}^{i}+\left\{1-F^{i-1}\left(X_{n-1}\right)\right\} \circ I_{n-1}^{i-1}-D_{n}^{i}, \\
i=2, \ldots, M .
\end{gathered}
$$

where " $\circ$ " is defined in (1.1) and $F^{i}\left(X_{n-1}\right) \circ I_{n-1}^{i}$ represents the number of items of quality $i$ that survived from period $n-1$ and $\left\{1-F^{i-1}\left(X_{n-1}\right)\right\} \circ I_{n-1}^{i-1}$ is the number of items of quality $i$ that moved to quality Class $i$ from Class $i-1$. The function $F^{i}\left(X_{n-1}\right)$ plays the same role as parameter $\alpha$ in (1.1). The dependence on $X_{n-1}$ is implicit here to stress the dependence of the process affecting the change of quality on the environment. Note we may write

$$
F^{i}=\left\{F^{i}\left(e_{1}\right), \ldots, F^{i}\left(e_{N}\right)\right\}:=\left\{F_{1}^{i}, \ldots, F_{N}^{i}\right\} .
$$

6. The process $\left\{X_{n}\right\}_{n \in \mathbb{N}}$ is a Markov chain with semimartingale representation (see [6])

$$
X_{n}=A X_{n-1}+M_{n}
$$

where $\left\{M_{n}\right\}_{n \in \mathbb{N}}$ is a martingale with respect to the complete filtration $\left\{\mathcal{F}_{n}\right\}_{n \in \mathbb{N}}$ generated by $\left\{X_{n}\right\}_{n \in \mathbb{N}}$ and $A$ denotes the transition probability matrix of the Markov chain $\left\{X_{n}\right\}$.

7. The accumulated losses up to time $n$ due to loss of qualities may be written as

$$
c_{n}=c_{n-1}+\sum_{i=1}^{M}\left\{1-F^{i}\left(x_{n-1}\right)\right\} \circ I_{n}^{i} \theta_{i} p_{n}, \quad n=1, \ldots,
$$

with $c_{0}=0$. 
Clearly, having an estimate of $\left(c_{n}, X_{n}\right)$ and a forecast of the quantity $\left(c_{n+1}, X_{n+1}\right)$ would be desirable in a sense that managers interested in stock control would have key information available to help in deciding the course of action to be taken on the number of items to stock.

The aim of this paper is to derive recursive expressions for the conditional distribution of $\left(c_{n}, X_{n}\right)$ and $\left(c_{n+1}, X_{n+1}\right)$ given the complete filtration $\left\{\mathcal{Y}_{n}\right\}_{n \in \mathbb{N}}$ generated by the observed processes $\left\{I_{n}^{i}\right\}_{n \in \mathbb{N}}, i=1, \ldots, M$ and $\left\{P_{n}\right\}_{n \in \mathbb{N}}$.

Inventory models for perishable items have been in the past, apart from [1, 2] and [3], examined from a different perspective. We are not aware of discrete models where items are allowed to lose quality. Also, interest in previous studies focused on investigating optimal operating characteristics (see [7, 8]).

In the next section, we derive a recursive conditional probability distribution of the cost process. In Section 3, a parameter updating algorithm is discussed.

\section{The Model}

Assume that all random variable are initially defined on an `ideal' probability space $(\Omega, \mathcal{F}, Q)$ such that:

1. The inventory levels $\left[I_{n}^{i}\right\}_{n \in \mathbb{N}}$ are $M$ sequences of independent random variables, with probability distributions $\left\{\Psi_{n}^{i}\right\}_{n \in \mathbb{N}}, i=1, \ldots, M$.

2. $\quad$ All other processes are defined in 1-7 of Section 1.

Let $\lambda_{0}=1$ and, for $k \geq 1$, define

$$
\begin{gathered}
\lambda_{k}=\frac{\Psi_{k}^{1}\left(F^{1}\left(X_{k-1}\right) \circ I_{k-1}^{1}-I_{k}^{1}+U_{k}\right)}{\Psi_{k}^{1}\left(I_{k}^{1}\right)} \\
\times \prod_{i=2}^{M} \frac{\Psi_{k}^{i}\left(F^{i}\left(X_{k-1}\right) \circ I_{k-1}^{i}+\left(1-F^{i-1}\left(X_{k-1}\right)\right) \circ I_{k-1}^{i-1}-I_{k}^{i}\right)}{\Psi_{k}^{i}\left(I_{k}^{i}\right)} .
\end{gathered}
$$

Set

$$
\Lambda_{n}=\prod_{k=0}^{n} \lambda_{k}
$$

Let $\{\mathcal{G}\}_{n \in \mathbb{N}}$ be the complete filtration generated by the processes $\left\{I_{n}\right\}_{n \in \mathbb{N}} ;\left\{P_{n}\right\}_{n \in \mathbb{N}}$; $\left\{D_{n}\right\}_{n \in \mathbb{N}} ;\left\{X_{n}\right\}_{n \in \mathbb{N}}$; and $\left\{Z_{n, l}^{i}\right\}_{n \in \mathbb{N}}, i=1, \ldots, M$, up to time $n$. Here

$Z_{n, l}^{i}$ refers to the state of the $l$ th item in the inventory at the end of period $n$. Thus,

$$
Z_{n, l}^{i}=\left\{\begin{array}{lc}
0, & \text { if the item is moved to class }(i+1) \\
1, & \text { otherwise }
\end{array}\right.
$$

It can be shown that the process $\left\{\Lambda_{n}\right\}_{n \in \mathbb{N}}$ is a $\{\mathcal{G}\}$-martingale.

Define a probability measure $P$ such that

$$
\Lambda_{n}=\left.\frac{d P}{d Q}\right|_{\mathcal{G}_{n}},
$$

where $\Lambda_{n}$ is given by (2.2). The existence of $P$ is guaranteed by Kolmogorov's Extension Theorem. 
The next lemma asserts that we can recover our original model under $P$.

Lemma 1: On $(\Omega, \mathcal{F})$ and under probability measure $P$, the demand processes $\left\{D_{n}^{i}\right\}$, $n=1,2, \ldots, \quad i=1, \ldots, M$, form sequence of random variables with probability distributions $\left\{\Psi_{n}^{i}\right\}_{n \in \mathbb{N}}$, where

$$
\begin{gathered}
D_{n}^{1}=F^{1}\left(X_{n-1}\right) \circ I_{n-1}^{1}+U_{n}-I_{n}^{1}, \\
D_{n}^{i}=F^{i}\left(X_{n-1}\right) \circ I_{n-1}^{i}+\left(1-F^{i-1}\left(X_{n-1}\right)\right) \circ I_{n-1}^{i-1}-I_{n}^{i}, i=2, \ldots, M .
\end{gathered}
$$

Proof: Let $g: \mathbb{Z}^{+} \rightarrow \mathbb{R}$ be a Borel test function and for $2 \leq k \leq M$, consider the expectation

$$
E_{P}\left[g\left(D_{n}^{k}\right) \mid \mathcal{G}_{n-1}\right]
$$

where $E_{P}(\cdot)$ is the expectation with respect to probability measure $P$.

Now, using the abstract Bayes Theorem (see [6]), expression (2.4) gives

$$
\begin{gathered}
E_{P}\left[g\left(D_{n}^{k}\right) \mid \mathcal{G}_{n-1}\right]=\frac{E_{Q}\left[g\left(D_{n}^{k}\right) \Lambda_{n} \mid \mathcal{G}_{n-1}\right]}{E_{Q}\left[\Lambda_{n} \mid \mathcal{G}_{n-1}\right]} \\
=\frac{E_{Q}\left[g\left(D_{n}^{k}\right) \lambda_{n} \mid \mathcal{G}_{n-1}\right]}{E_{Q}\left[\lambda_{n} \mid \mathcal{G}_{n-1}\right]}:=\frac{N}{D}
\end{gathered}
$$

where $\lambda_{n}$ and $\Lambda_{n}$ are given by (2.1) and (2.2), respectively. Using (2.1) we have

$$
\begin{gathered}
D=E_{Q}\left[\frac{\Psi_{n}^{1}\left(F^{1}\left(X_{n-1} 0 \circ I_{n-1}^{1}-I_{n}^{1}+U_{n}\right)\right.}{\Psi_{n}^{1}\left(I_{n}^{1}\right)}\right. \\
\left.\times \prod_{i=2}^{M} \frac{\Psi_{n}^{i}\left(F^{i}\left(x_{n-1}\right) \circ I_{n-1}^{i}-\left(1-F^{i-1}\left(X_{n-1}\right)\right) \circ I_{n-1}^{i-1}-I_{n}^{i}\right)}{\Psi_{n}^{i}\left(I_{n}^{i}\right)} \mid \mathcal{G}_{n-1}\right] \\
=\sum_{z^{1}, \ldots, z^{M}} \Psi_{n}^{1}\left(F^{1}\left(X_{n-1} \circ I_{n-1}^{1}-z^{1}+U_{n}\right)\right. \\
\times \prod_{I=2}^{M} \Psi_{n}^{i}\left(F^{i}\left(X_{n-1}\right) \circ I_{n-1}^{i}-\left(1-F^{i-1}\left(X_{n-1}\right)\right) \circ I_{n-1}^{i-1}-z^{i}\right)=1 .
\end{gathered}
$$

The numerator $N$ of (2.5) is equal to

$$
E_{Q}\left[g\left(F^{k}\left(X_{n-1}\right) \circ I_{n-1}^{k}-\left(1-F^{k-1}\left(X_{n-1}\right)\right) \circ I_{n-1}^{k 01}-I_{n}^{i}\right) \lambda_{n} \mid \mathcal{G}_{n-1}\right] .
$$

Again, using (2.1), the above is

$$
E_{Q}\left[g \left(F^{k}\left(X_{n-1} \circ I_{n-1}^{k}-\left(1-F^{k-1}\left(X_{n-1}\right)\right) \circ I_{n-1}^{k-1}-I_{n}^{k}\right)\right.\right.
$$




$$
\begin{gathered}
\times \frac{\Psi_{n}^{1}\left(F^{1}\left(X_{n-1}\right) I_{n-1}^{1}-I_{n}^{1}+U_{n}\right)}{\Psi_{n}^{1}\left(I_{n}^{1}\right)} \\
\left.\times \prod_{i=2}^{M} \frac{\Psi_{n}^{i}\left(F^{i}\left(X_{n-1}\right) \circ I_{n-1}^{i}-\left(1-F^{i-1}\left(X_{n-1}\right)\right) \circ I_{n-1}^{i-1}-I_{n}^{i}\right)}{\Psi_{n}^{i}\left(I_{n}^{i}\right)} \mid \mathcal{G}_{n-1}\right] \\
=E_{Q}\left[\frac{\Psi_{n}^{1}\left(F^{1}\left(X_{n-1}\right) \circ I_{n}^{1}-I_{n}^{1}+U_{n}\right)}{\Psi_{n}^{1}\left(I_{n}^{1}\right)}\right. \\
\times \prod_{i=2}^{M} \frac{\Psi_{n}^{i}\left(F^{i}\left(X_{n-1}\right) \circ I_{n-1}^{i}-\left(1-F^{i-1}\left(X_{n-1}\right)\right) \circ I_{n-1}^{i-1}-I_{n}^{i}\right)}{\Psi_{n}^{i}\left(I_{n}^{i}\right)} \\
\times E_{Q}\left[g\left(F^{k}\left(X_{n-1}\right) \circ I_{n-1}^{k}-\left(1-F^{k-1}\left(X_{n-1}\right)\right) \circ I_{n-1}^{k-1}-I_{n}^{k}\right)\right. \\
\left.\times \frac{\Psi_{n}^{k}\left(F^{k}\left(X_{n-1}\right) \circ I_{n-1}^{k}+\left(1-F^{k-1}\left(X_{n-1}\right)\right) \circ I_{n-1}^{k-1}-I_{n}^{k}\right)}{\Psi_{n}^{k}\left(I_{n}^{k}\right)}\left|I_{n}^{i}, i \neq k, \mathcal{G}_{n-1}\right| \mathcal{G}_{n-1}\right] .
\end{gathered}
$$

The inner expectation in the above relation is equal to

$$
\begin{gathered}
\sum_{z^{k}} g\left(F^{k}\left(X_{n-1}\right) \circ I_{n-1}^{k}-\left(1-F^{k-1}\left(X_{n-1}\right)\right) \circ I_{n-1}^{k-1}-z^{k}\right) \\
\times \frac{\Psi_{n}^{k}\left(F^{k}\left(X_{n-1}\right) \circ I_{n-1}^{k}+\left(1-F^{k-1}\left(X_{n-1}\right)\right) \circ I_{n-1}^{k-1}-z^{k}\right)}{\Psi_{n}^{k}\left(z^{k}\right)} \\
=\sum_{u^{k}} g\left(u^{k}\right) \Psi^{k}\left(u^{k}\right) .
\end{gathered}
$$

It is easily seen that the expectation with respect to $Q$ of the remaining factors in (2.6) is 1 from which the lemma follows.

Remark: Note that (1.3) and (1.4) are unchanged with respect to probability measure $Q$.

Recall that the aim of the paper is to derive the joint distribution of $\left(c_{n}, X_{n}\right)$. For that, consider $E_{P}\left[\left\langle X_{n}, e_{j}\right\rangle g\left(c_{n}\right) \mid \mathcal{Y}_{n}\right]$ where $g$ is an arbitrary Borel test function. Here $\langle\cdot, \cdot\rangle$ denotes the inner product in $\mathbb{R}^{N}$.

Again, using the abstract Bayes Theorem (see [6]), we have

$$
E_{P}\left[\left\langle X_{n}, e_{j}\right\rangle g\left(c_{n}\right) \mid \mathcal{Y}_{n}\right]=\frac{E_{Q}\left[\left\langle X_{n}, e_{j}\right\rangle g\left(c_{n}\right) \Lambda_{n} \mid \mathcal{Y}_{n}\right]}{E_{Q}\left[\Lambda_{n} \mid \mathcal{Y}_{n}\right]} .
$$

The numerator in (2.7) represents the unnormalized conditional expectation. Write

$$
E_{Q}\left[\left\langle X_{n}, e_{j}\right\rangle g\left(c_{n}\right) \Lambda_{n} \mid \mathcal{Y}_{n}\right]:=\sum_{c} g(c) q_{n}^{j}(c) .
$$


This is a measure-valued process where the normalizing denominator in (2.7) is simply

$$
E_{Q}\left[\Lambda_{n} \mid \mathcal{Y}_{n}\right]=\sum_{k} \sum_{l=1}^{N} q_{n}^{l}(k)
$$

Theorem 1: The value of $q_{n}^{j}(\cdot)$ is given by the recursion

$$
\begin{gathered}
q_{n}^{j}=\sum_{k_{1}=0}^{I_{n}^{1}} \ldots \sum_{k_{M}=0}^{I_{n}^{M}} \sum_{l_{1}=1}^{I_{n-1}^{1}} \ldots \sum_{l_{M}}^{I_{n-1}^{M}} \sum_{r=0}^{N} A_{j r} \frac{\Psi_{n}^{1}\left(l_{1}-I_{n}^{1}+U_{n}\right)}{\Psi_{n}^{1}\left(I_{n}^{1}\right)} \\
\times \prod_{i=2}^{M} \frac{\Psi_{n}^{i}\left(l_{i}-I_{n-1}^{i-1} k-l_{i-1}-I_{n}^{i}\right)}{\Psi_{n}^{i}\left(I_{n}^{i}\right)} \prod_{i=1}^{M}\left(\begin{array}{c}
I_{n}^{i} \\
k_{i}
\end{array}\right)\left(\begin{array}{c}
I_{n-1}^{i} \\
l_{i}
\end{array}\right)\left(F_{r}^{i}\right)^{l_{i}}\left(1-F_{r}^{i}\right)^{I_{n-1}^{i}-l_{i}} \\
\times\left(1-F_{j}^{i}\right)^{k_{i}}\left(F_{j}^{i}\right)^{I_{n}^{i}-k_{i}} q_{n-1}^{r}\left(c-\sum_{i=1}^{M} k_{i} \theta_{i} p_{n}\right),
\end{gathered}
$$

with normalized form $p_{n}^{j}(c)=\frac{q_{n}^{j}(c)}{\sum_{k} \sum_{l}^{l}(k)}$.

Also, the marginal distribution of the cost is $p_{n}(c)=\sum_{j=1}^{N} p_{n}^{j}(c)$.

Proof: In view of (1.4), (2.1) and (2.2) and for any test function $g$, expression (2.8) is equal to

$$
\begin{aligned}
& \sum_{r=1}^{N} A_{j r} E_{Q}\left[\left\langle X_{n-1}, e_{r}\right\rangle g\left\{c_{n-1}+\sum_{i=1}^{M}\left(1-F_{j}^{i}\right) \circ I_{n}^{i} \theta_{i} p_{n}\right\} \Lambda_{n-1} \lambda_{n} \mid \mathcal{Y}_{n}\right] \\
& =\sum_{r=1}^{N} \sum_{k_{1}=0}^{I_{n}^{1}} \ldots \sum_{k_{M}=0}^{I_{n}^{M}} \sum_{l_{1}=1}^{I_{n-1}^{1}} \ldots \sum_{l_{M}}^{I_{n-1}^{M}} \sum_{r=0}^{N} A_{j r} \frac{\Psi_{n}^{1}\left(l_{1}-I_{n}^{1}+U_{n}\right)}{\Psi_{n}^{1}\left(I_{n}^{1}\right)} \\
& \times \prod_{i=2}^{M} \frac{\Psi_{n}^{i}\left(l_{i}-I_{n-1}^{i-1} k-l_{i-1}-I_{n}^{i}\right)}{\Psi_{n}^{i}\left(I_{n}^{i}\right)} \prod_{i=1}^{M}\left(\begin{array}{c}
I_{n}^{i} \\
k_{i}
\end{array}\right)\left(\begin{array}{c}
I_{n-1}^{i} \\
l_{i}
\end{array}\right)\left(F_{r}^{i}\right)^{l_{i}}\left(1-F_{r}^{i}\right)^{I_{n-1}^{i}-l_{i}} \\
& \times\left(1-F_{j}^{i}\right)^{k_{i}}\left(F_{j}^{i}\right)^{I_{n}^{i}-k_{i}} E_{Q}\left[\left\langle X_{n-1}, e_{r}\right\rangle g\left(c_{n-1}+\sum_{i=1}^{M} k_{i} \theta_{i} p_{n}\right) \mid \mathcal{Y}_{n-1}\right] \\
& =\sum_{r=1}^{N} \sum_{k_{1}=0}^{I_{n}^{1}} \ldots \sum_{k_{M}=0}^{I_{n}^{M}} \sum_{l_{1}=1}^{I_{n-1}^{1}} \ldots \sum_{l_{M}}^{I_{n-1}^{M}} \sum_{r=0}^{N} A_{j r} \frac{\Psi_{n}^{1}\left(l_{1}-I_{n}^{1}+U_{n}\right)}{\Psi_{n}^{1}\left(I_{n}^{1}\right)}
\end{aligned}
$$




$$
\begin{aligned}
& \times \prod_{i=2}^{M} \frac{\Psi_{n}^{i}\left(l_{i}+I_{n-1}^{i-1}-l_{i-1}-I_{n}^{i}\right)}{\Psi_{n}^{i}\left(I_{n}^{i}\right)} \prod_{i=1}^{M}\left(\begin{array}{c}
I_{n}^{i} \\
k_{i}
\end{array}\right)\left(\begin{array}{c}
I_{n-1}^{i} \\
l_{i}
\end{array}\right)\left(F_{r}^{i}\right)^{l_{i}}\left(1-F_{r}^{i}\right)^{I_{n-1}^{i}-l_{i}} \\
& \quad \times\left(1-F_{j}^{i}\right)^{k_{i}}\left(F_{j}^{i}\right)^{I_{n}^{i}-k_{i}} \sum_{u} g\left(u+\sum_{i=1}^{M} k_{i} \theta_{i} p_{n}\right) \mid q_{n-1}^{r}(u) \\
& =\sum_{r=1}^{N} \sum_{k_{1}=0}^{I_{n}^{1}} \ldots \sum_{k_{M}=0}^{I_{n}^{M}} \sum_{l_{1}=1}^{I_{n-1}^{1}} \ldots \sum_{l_{M}}^{I_{n-1}^{M}} \sum_{r=0}^{N} A_{j r} \frac{\Psi_{n}^{1}\left(l_{1}-I_{n}^{1}+U_{n}\right)}{\Psi_{n}^{1}\left(I_{n}^{1}\right)} \\
& \times \prod_{i=2}^{M} \frac{\Psi_{n}^{i}\left(l_{i}+I_{n-1}^{i-1}-l_{i-1}-I_{n}^{i}\right)}{\Psi_{n}^{i}\left(I_{n}^{i}\right)} \prod_{i=1}^{M}\left(\begin{array}{c}
I_{n}^{i} \\
k_{i}
\end{array}\right)\left(\begin{array}{c}
I_{n-1}^{i} \\
l_{i}
\end{array}\right)\left(F_{r}^{i}\right)^{l_{i}}\left(1-F_{r}^{i}\right)^{I_{n-1}^{i}-l_{i}} \\
& \quad \times\left(1-F_{j}^{i}\right)^{k_{i}}\left(F_{j}^{i}\right)^{I_{n}^{i}-k_{i}} \sum_{c} g(c) q_{n=1}^{r}\left(c-\sum_{i=1}^{M} k_{i} \theta_{i} p_{n}\right) .
\end{aligned}
$$

This holds for all test functions $g$. So the recursion for $q_{n}^{j}$ in the theorem is true.

Now, we shall be interested in the joint distribution of $\left(c_{n+1}, X_{n+1}\right)$ given the information accumulated up to time $n$. That is, we wish to predict the behavior of $(c, X)$ one period of the future. Needless to say, this can be helpful for planning purposes of inventory management.

Consider the unnormalized conditional expectation $\rho_{n, n+1}(\cdot)$ such that

$$
E_{Q}\left[\left\langle X_{n+1}, e_{m}\right\rangle g\left(c_{n+1}\right) \Lambda_{n+1} \mid \mathcal{Y}_{n}\right]=\sum_{c} g(c) \rho_{n, n+1}^{m}(c)
$$

Then, we have

Theorem 2: The value of $\rho_{n, n+1}^{m}(\cdot)$ is given by the recursion:

$$
\begin{gathered}
\rho_{n, n+1}^{m}(c)=\sum_{h_{1} \geq 1} \ldots \sum_{h_{M} \geq 1} \sum_{k_{1}=0}^{h_{1}} \ldots \sum_{k_{M}=0}^{h_{M}} \sum_{I_{1}=0}^{I_{n}^{1}} \ldots \sum_{I_{M}=0}^{I_{n}^{M}} \sum_{j=1}^{N} A_{m j} \Psi_{n+1}^{1}\left(l_{1}-h_{1}+u_{n+1}\right) \\
\times \prod_{i=2}^{M} \Psi_{n+1}^{i}\left(l_{i}+I_{n}^{i-1}-l_{i-1}-h_{i}\right) \prod_{i=1}^{M}\left(\begin{array}{c}
h_{i} \\
k_{i}
\end{array}\right)\left(\begin{array}{c}
I_{n}^{i} \\
l_{i}
\end{array}\right)\left(F_{j}^{i}\right)^{l_{i}}\left(1-F_{j}^{i}\right)^{I_{n}^{i}-l_{i}} \\
\times\left(1-F_{m}^{i}\right)^{h_{i}}\left(F_{m}^{i}\right)^{h_{i}-k_{i}} q_{n}^{j}\left(c-\sum_{i=1}^{M} k_{i} \theta_{i} P_{n+1}\right),
\end{gathered}
$$

where $q_{n}^{j}(\cdot)$ is given recursively in Theorem 1 .

Proof: The left-hand side of (2.9) is equal to 


$$
\sum_{j} A_{m j} E_{Q}\left[\left\langle X_{n}, e_{j}\right\rangle g\left(c_{n}+\sum_{i=1}^{M}\left(1-F_{m}^{i}\right) \circ I_{n+1}^{i} \theta_{i} p_{n}\right) \Lambda_{n} \lambda_{n+1} \mid \mathcal{Y}_{n}\right]
$$

Note that $I_{n+1}^{i}, i=1, \ldots, M$ are known at time $n$. So to get the result, use the fact that under probability measure $Q$, they are sequences of independent random variables with probability distribution $\Psi_{n+1}^{i}$, respectively. This completes the proof.

It is worth noting at this stage that the estimates obtained in Theorems 2 and 3 are optimal in the sense that they minimize the mean squares of the errors. For more details on similar estimates, see [5].

\section{Parameter Estimation}

This section is concerned with estimating the parameters

$$
\theta=\left\{F_{j}^{i}, A_{s r}, \Psi^{i}(d), i=1, \ldots, M ; j, s, r=1, \ldots, N ; 0 \leq d \leq D\right\} .
$$

Here, we assume for simplicity that the marginal distribution $\Psi^{i}$ of the demand $D^{i}$ has finite support and that $P\left[D_{n}^{i}>D\right]=0$ for all $n=1, \ldots$ and $i=1, \ldots, M$.

Recall that initially we assumed that we have an 'ideal' probability space $(\Omega, \mathcal{F}, P)$. We shall denote $P$ by $P_{\theta}$ to emphasize the dependence of $P$ on $\theta$.

Our estimation procedure is based on the (EM) algorithm (see [5]). The method starts with a guess (prior) value of $\theta$. Then this value is updated at each time $n$ based on the information gathered in the $\sigma$-field generated by the inventory levels and the prices.

Let

$$
\widehat{\theta}=\left\{\widehat{F}_{j}^{i}, \widehat{A}_{s r}, \widehat{\Psi}^{i}(d), i=1, \ldots, M ; j, s, r=1, \ldots, N ; 0 \leq d \leq D\right\} .
$$

In order to update $\theta$ to $\widehat{\theta}$ at time $n$, define the martingale

$$
\begin{aligned}
& \Gamma_{n}=\prod_{k=1}^{n} \prod_{i=1}^{M} \prod_{l=1}^{L_{n}} \prod_{j=1}^{N}\left(\frac{\widehat{F}_{j}^{i}}{i^{j}}\right)^{I\left(Z_{k l}^{i}=1\right)}\left(\frac{1-\widehat{F}_{j}^{i}}{1-F_{j}^{i}}\right)^{I\left(Z_{k, l}^{i}=0\right)} \\
& \times \prod_{r s=1}^{N}\left(\frac{\widehat{A}_{s r}}{A_{s r}}\right)^{I\left(X_{k-1}=e_{r}, X_{k}=e_{s}\right)} \prod_{i=1}^{M} \prod_{d=0}^{D}\left(\frac{\widehat{\Psi}^{i}(d)}{\Psi^{i}(d)}\right)^{I\left(D_{k}^{i}=d\right)} .
\end{aligned}
$$

It can be shown that $E_{P_{\theta}}\left[\Gamma_{n}\right]=1$ so that we can set

$$
\left.\frac{d P_{\widehat{\theta}}}{d P_{\theta}}\right|_{\mathcal{G}_{n}}=\Gamma_{n}
$$

Let

$$
\mathcal{H}_{n}=\mathcal{Y}_{n} \vee \sigma\left\{D_{k}^{i}, i=1, \ldots, M, k \leq n\right\}
$$

and 


$$
\widehat{E}[\cdot]:=E_{P_{\hat{\theta}}}[\cdot] .
$$

Then it follows from (3.1) and (3.2) that

$$
\begin{gathered}
\widehat{E}\left[\operatorname{og} \Gamma_{n} \mid \mathcal{H}_{n}\right]=\sum_{j=1}^{N} \sum_{i=1}^{N} \widehat{E}\left[\sum_{k=1}^{n} \sum_{l=1}^{L_{k}} I\left(Z_{k, l}^{i}=1\right) \mid \mathcal{H}_{n}\right] \log \widehat{F}_{j}^{i} \\
+\sum_{j=1}^{N} \sum_{i=1}^{N} \widehat{E}\left[\sum_{k=1}^{n} \sum_{l=1}^{L_{k}} I\left(Z_{k, l}^{i}=0\right) \mid \mathcal{H}_{n}\right] \log \left(1-\widehat{F}_{j}^{i}\right) \\
+\sum_{j=1}^{N} \sum_{i=1}^{N} \widehat{E}\left[\sum_{k=1}^{n} \sum_{l=1}^{L_{k}}\left\langle X_{k-1}, e_{r}\right\rangle\left\langle X_{k}, e_{s}\right\rangle \mid \mathcal{H}_{n}\right] \log \widehat{A}_{s r} \\
+\sum_{i=1}^{M} \sum_{d=0}^{D} \sum_{k=1}^{n} I\left(D_{n}^{k}=d\right) \log \widehat{\Psi}^{i}(d)+R(\theta)
\end{gathered}
$$

where $R(\theta)$ is an expression which does not depend on $\widehat{\theta}(n)$.

Write

$$
\begin{gathered}
Z_{n}^{i}:=\sum_{k=1}^{n} \sum_{l=1}^{L_{k}} I\left(Z_{k, l}^{i}=1\right), \\
Z_{n}^{i+1}:=\sum_{k=1}^{n} \sum_{l=1}^{L_{k}} I\left(Z_{k, l}^{i}=0\right), \\
J_{n}^{s r}:=\sum_{k=1}^{n}\left\langle X_{k-1}, e_{r}\right\rangle\left\langle X_{k}, e_{s}\right\rangle \\
\xi_{n}^{\alpha}\left(Z_{n}^{i}\right):=E_{Q}\left[Z_{n}^{i}\left\langle X_{k}, e_{\alpha}\right\rangle \Lambda_{n} \mid \mathcal{H}_{n}\right], \\
\xi_{n}\left(Z_{n}^{i}\right):=\sum_{\alpha=1}^{N} \xi_{n}^{\alpha}\left(Z_{n}^{i}\right)=E_{Q}\left[Z_{n}^{i} \Lambda_{n} \mid \mathcal{H}_{n}\right] \\
q_{n}^{\alpha}:=e_{Q}\left[\left\langle X_{k}, e_{\alpha}\right\rangle \Lambda_{n} \mid \mathcal{H}_{n}\right]
\end{gathered}
$$




$$
\begin{gathered}
q_{n}\left(J_{n}^{s r} X_{n}\right):=E_{Q}\left[J_{n}^{s r} \Lambda_{n} X_{n} \mid \mathcal{H}_{n}\right] \\
q_{n}\left(J_{n}^{s r}\right):=\left\langle q_{n}\left(J_{n}^{s r} X_{n}\right),(1,1, \ldots, 1)\right\rangle .
\end{gathered}
$$

Maximizing the conditional log-likelihood in (3.3) with respect to $\widehat{\theta}$, we obtain the following result.

Theorem 2:

$$
\begin{gathered}
\widehat{\Psi}^{i}(d)=\frac{\sum_{k=1}^{n} I\left(D_{k}^{i}=d\right)}{n}, \\
\widehat{F}_{j}^{i}(n)=\frac{E\left[Z_{n}^{i} \mid \mathcal{H}_{n}\right]}{E\left[Z_{n}^{i} \mid \mathcal{H}_{n}\right]+E\left[Z_{n}^{i+1} \mid \mathcal{H}_{n}\right]}=\frac{\xi_{n}\left(Z_{n}^{i}\right)}{i_{n}\left(Z_{n}^{i}\right)+\xi_{n}\left(Z_{n}^{i+1}\right)} \\
\widehat{A}_{s r}^{n}=\frac{q_{n}\left(J_{n}^{s r}\right)}{\sum_{s=1}^{N} q_{n}\left(J_{n}^{s r}\right)}
\end{gathered}
$$

and the unnormalized conditional probability distribution of $\xi_{n}\left(Z_{n}^{i}\right) ; \xi_{n}\left(Z_{n}^{i+1}\right)$ and $q_{n}\left(J_{n}^{s r}\right)$ are given by the following recursions:

$$
\begin{gathered}
\xi_{n}\left(Z_{n}^{i}\right)=\sum_{\alpha=1}^{N} \xi_{n}^{\alpha}\left(Z_{n}^{i}\right), \\
\xi_{n}^{\alpha}\left(Z_{n}^{i}\right)=I_{n}^{i} q_{n}^{\alpha}+\sum_{\beta=1}^{N} \xi_{n-1}^{\beta}\left(Z_{n-1}^{i}\right) \lambda_{n}(\beta) A_{\beta \alpha},
\end{gathered}
$$

where

$$
\begin{gathered}
\lambda_{n}(\beta)=\frac{\Psi_{n}^{1}\left(F_{\beta}^{1} \circ I_{n-1}^{1}-I_{n}^{1}+u_{n}\right)}{\Psi_{n}^{1}\left(I_{n}^{1}\right)} \prod_{i=2}^{M} \frac{\Psi_{n}^{i}\left(F_{\beta}^{i} \circ I_{n-1}^{i}-\left(1-F_{\beta}^{i-1}\right) \circ I_{n-1}^{i-1}-I_{n}^{i}\right)}{\Psi_{n}^{1}\left(I_{n}^{i}\right)} \\
q_{n}\left(J_{n}^{s r} X_{n}\right)=\sum_{\beta=1}^{N}\left\langle q_{n-1}\left(J_{n-1}^{s r}, X_{n-1}\right), e_{\beta}\right\rangle A_{e_{\beta}} \lambda_{n}(\beta)+A_{r s} e_{s} q_{n-1}^{r} \lambda_{n}(r), \\
q_{n-1}^{r}=E_{Q}\left[\left\langle X_{n-1}, e_{r}\right\rangle \Lambda_{n-1} \mid \mathcal{H}_{n-1}\right] .
\end{gathered}
$$

Proof: Straightforward algebra gives (3.4)-(3.6). To derive (3.7), write

$$
Z_{n}^{i}=Z_{n-1}^{i}+\sum_{l=1}^{L_{n}} I\left(Z_{n, l}^{i}=1\right)=Z_{n-1}^{i}+I_{n}^{i}
$$

Hence,

$$
\begin{gathered}
\xi_{n}^{\alpha}\left(Z_{n}^{i}\right)=E_{Q}\left[Z_{n}^{i}\left\langle X_{n}, e_{\alpha}\right\rangle \Lambda_{n} \mid \mathcal{H}_{n}\right] \\
=E_{Q}\left[Z_{n-1}^{i}\left\langle A X_{n-1}, e_{\alpha}\right\rangle \Lambda_{n-1} \lambda_{n} \mid \mathcal{H}_{n}\right]+I_{n}^{i} E_{Q}\left[\left\langle X_{n}, e_{\alpha}\right\rangle \Lambda_{n} \mid \mathcal{H}_{n}\right]
\end{gathered}
$$


The second expression in (3.8) is $I_{n}^{i} q_{n}^{\alpha}$. The scalar product in the first expression in (3.8) is equal to $\sum_{\beta}\left\langle X_{n-1}, e_{\beta}\right\rangle A_{\beta \alpha}$, which leads to

$$
\begin{gathered}
\xi_{n}^{\alpha}\left(Z_{n}^{i}\right)=\sum_{\beta=1}^{N} A_{\beta \alpha} \lambda_{n}(\beta) E_{Q}\left[\left\langle X_{n-1}, e_{\beta}\right\rangle Z_{n-1}^{i} \Lambda_{n-1} \mid \mathcal{H}_{n-1}\right]+I_{n}^{i} q_{n}^{\alpha} \\
=\sum_{\beta=1}^{N} A_{\beta \alpha} \lambda_{n}(\beta) \xi_{n-1}^{\beta}\left(Z_{n-1}^{i}\right)+I_{n}^{i} q_{n}^{\alpha}
\end{gathered}
$$

which is expression (3.7). This completes the proof.

\section{Summary}

In this paper, we proposed another version of a discrete-time, discrete-state inventory model for items of changing quality. The quality alteration of the items is affected by random changes in the ambient environment. Also, prices of the items of different quality are allowed go change from period to period in a random fashion. Based on observing the history of the inventory level and prices, recursive estimators as well as predictors for the joint distribution of the accumulated losses and the state of the environment were derived. Further, parameters estimation were discussed.

\section{References}

[1] Aggoun, L., Benkherouf, L., and Tadj, L., A hidden Markov model for an inventory system with perishable items, J. of Applied Math. and Stoch. Anal. 10:4 (1997), 423430.

[2] Aggoun, L., Benkherouf, L., and Tadj, L., A stochastic jump inventory model with deteriorating items, Stoch. Anal. and Appl., to appear.

[3] Aggoun, L., Benkherouf, L., and Tadj, L., Optimal adaptive estimators for partially observed numbers of defective items in inventory models, Math. and Comp. Modeling 29 (1999), 83-93.

[4] Aggoun, L., Benkherouf, L., and Tadj, L., A stochastic inventory model with perishable and aging items, J. of Appl. Math. and Stoch. Anal. 12:1 (1999), 23-29.

[5] Baum, L.E. and Petrie, T., Statistical inference for probabilistic functions of finite state Markov chains, Annals of the Inst. of Stat. Math. 37 (1966), 1554-1563.

[6] Elliot, R.J., Aggoun, L., and Moore, J.B., Hidden Markov Models: Estimation and Control, Springer-Verlag, New York 1995.

[7] Nahmias, S., Perishable inventory theory: A review, Oper. Res. 30 (1982), 680-707.

[8] Rafaat, F., Survey of literature on continuously deteriorating inventory models, J. of Oper. Res. Soc. 42 (1991), 27-37. 


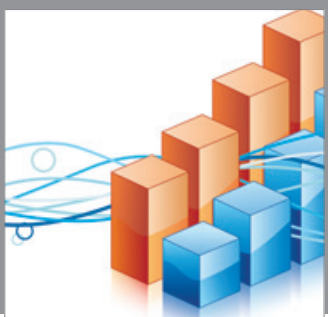

Advances in

Operations Research

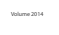

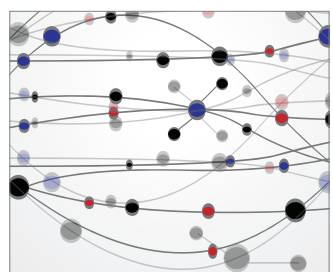

\section{The Scientific} World Journal
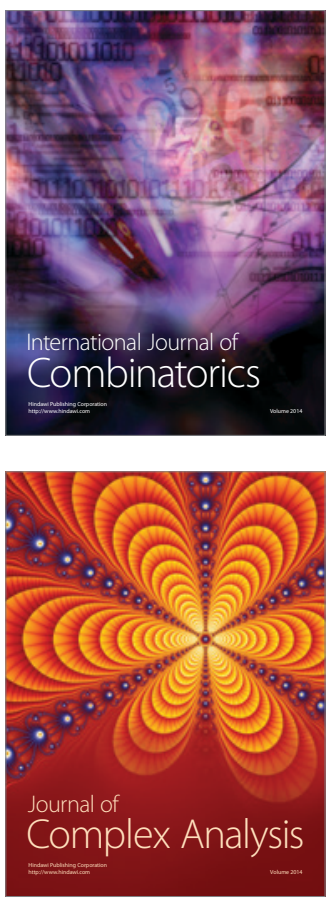

International Journal of

Mathematics and

Mathematical

Sciences
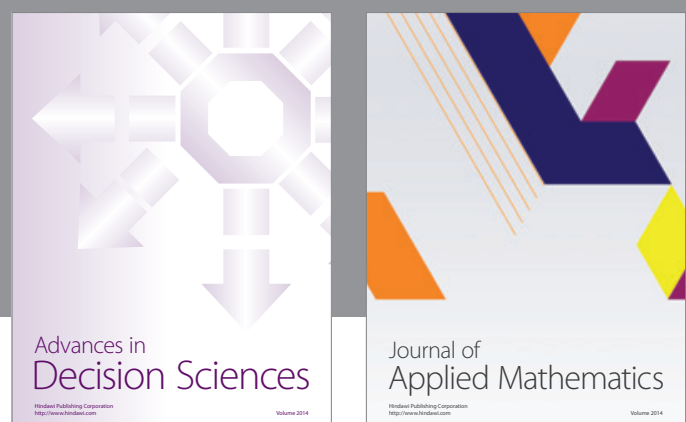

Journal of

Applied Mathematics
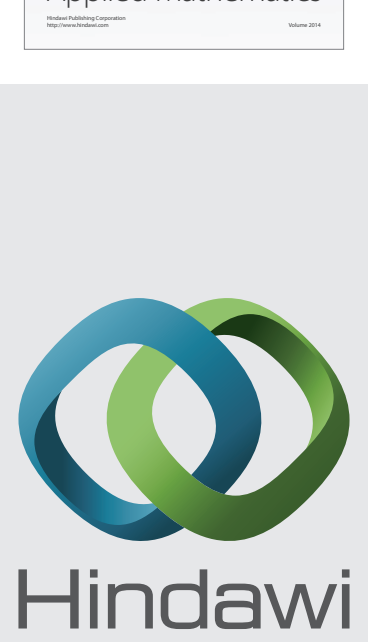

Submit your manuscripts at http://www.hindawi.com
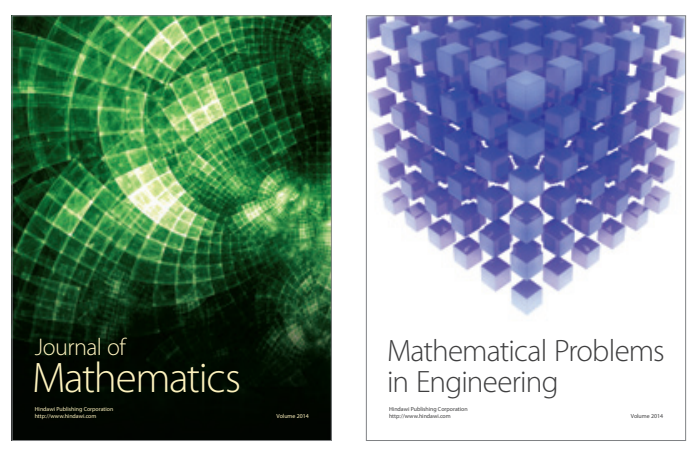

Mathematical Problems in Engineering
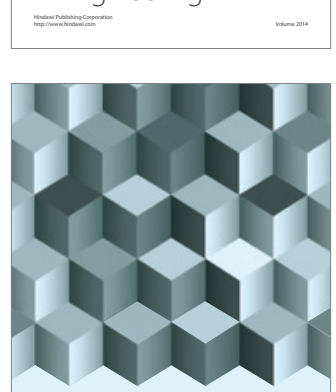

Journal of

Function Spaces
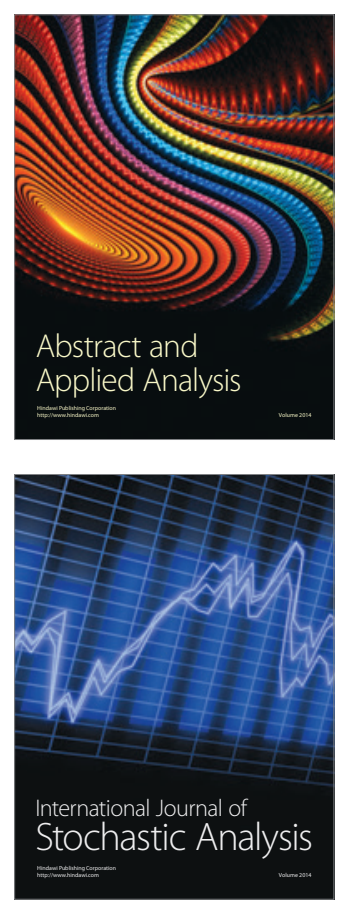

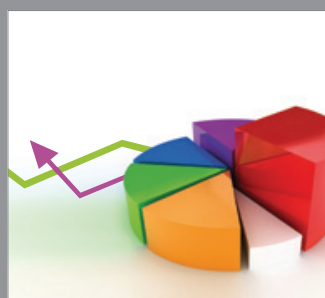

ournal of

Probability and Statistics

Promensencen
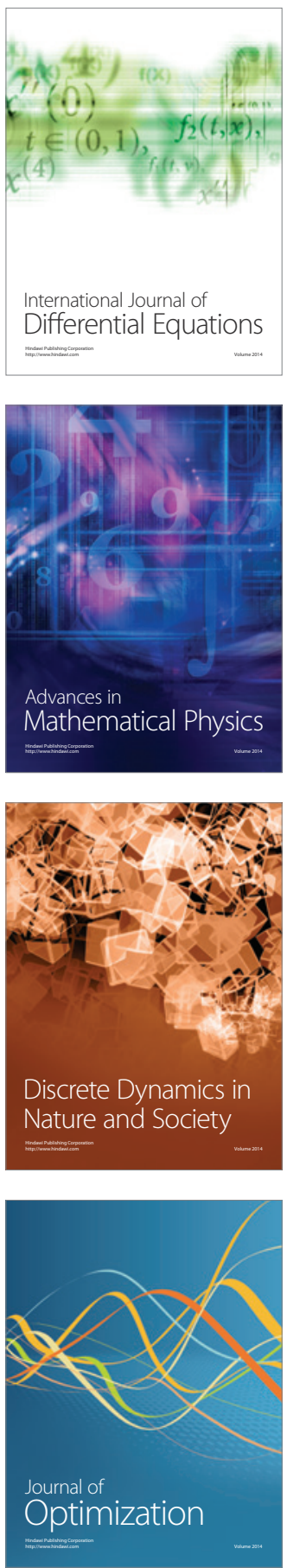